\title{
Image Synthesis from a Single Example Image
}

\author{
Thomas Vetter ${ }^{1}$ and Tomaso Poggio ${ }^{2}$ \\ 1 Max-Planck-Institut für biologische Kybernetik, \\ Spemannstr.38 D-72076 Tübingen, Germany \\ Email: vetter@mpik-tueb.mpg.de \\ 2 Center for Biological and Computational Learning \\ M.I.T. Cambridge, MA 02139 U.S.A. \\ Email:poggio@ai.mit.edu
}

\begin{abstract}
The need to generate new views of a $3 \mathrm{D}$ object from a single real image arises in several fields, including graphics and object recognition. While the traditional approach relies on the use of 3D models, we exploit 2D image transformations that are specific to the relevant object class and learnable from example views of other "prototypical" objects of the same class.

For linear object classes we show that linear transformations can be learned exactly from a basis set of $2 \mathrm{D}$ prototypical views. We demonstrate the approach on artificial objects and then show preliminary evidence that the technique can effectively "rotate" high-resolution face images from a single $2 \mathrm{D}$ view.
\end{abstract}

Index Items - 3D Object recognition, rotation invariance, deformable models, image synthesis

\section{Introduction}

View-based approaches to $3 \mathrm{D}$ object recognition and graphics may avoid the explicit use of $3 \mathrm{D}$ models by exploiting the memory of several views of the object and the ability to interpolate or generalize among them. In many situations however a sufficient number of views may not be available. In an extreme case we may have to do this with only one real view. Consider for instance the problem of recognizing a specific human face under a different pose or expression when only one example picture is given. Our visual system is certainly able to perform this task [1]. The obvious explanation is that we exploit prior information about how face images transform, learned through extensive experience with other faces. Thus the idea (see [2]), is to learn class-specific image-plane transformations from examples of objects of the same class and then to apply them to the real image of the new object in order to synthesize virtual views.

The work described in this paper is based on the idea of linear object classes. These are $3 \mathrm{D}$ objects whose $3 \mathrm{D}$ shape can be represented as a linear combination of a sufficiently small number of prototypical objects. Linear object classes have the properties that new orthographic views of any object of the class under uniform affine $3 D$ transformations, and in particular rigid transformations in 
$3 \mathrm{D}$, can be generated exactly if the corresponding transformed views are known for the set of prototypes. Thus if the training set consist of frontal and rotated views of a set of prototype faces, any rotated view of a new face can be generated from a single frontal view - provided that the linear class assumption holds.

Key to our approach is a representation of an object view in terms of a shape vector and texture vector (see also Jones and Poggio [3] and Beymer and Poggio [4]). The first gives the image-plane coordinates of feature points of the object surface; the second provides their color or grey-level. Assuming correspondence, we will represent an image as follows: we code its $2 \mathrm{D}$-shape as the deformation field of selected feature points - each corresponding in the limit to a pixel - from a reference image in the same pose, which serves as the origin of our coordinate system. The texture is coded as the intensity map of the image with feature points set in correspondence with the reference image. Thus each component of the shape and the texture vector refers to the same feature point e.g. pixel. Notice, we do not need correspondence between different poses as in the parallel deformation technique of Poggio and Brunelli [5] and Beymer et al.[6].

\section{Linear Object Classes}

\subsection{Shape of $3 \mathrm{D}$ objects}

Consider a 3D view of an three-dimensional object, which is defined in terms of pointwise features [2]. A $3 \mathrm{D}$ view can be represented by a vector $\mathbf{X}=$ $\left(x_{1}, y_{1}, z_{1}, x_{2}, \ldots, y_{n}, z_{n}\right)^{T}$, that is, by the $x, y, z$-coordinates of its $n$ feature points (we factor out translation). Assume that $\mathbf{X} \in \Re^{3 n}$ is the linear combination of $q 3 \mathrm{D}$ views $\mathbf{X}_{i}$ of other objects of the same dimensionality, such that:

$$
\mathbf{X}=\sum_{i=1}^{q} \alpha_{i} \mathbf{X}_{i}
$$

$\mathbf{X}$ is then the linear combination of $q$ vectors in a $3 n$ dimensional space, each vector representing an object of $n$ pointwise features. Consider now the linear operator $L$ associated with a desired uniform transformation such as for instance a specific rotation in $3 \mathrm{D}$. Let us define $\mathbf{X}^{r}=L \mathbf{X}$ the rotated $3 \mathrm{D}$ view of object $\mathbf{X}$. Because of the linearity of the group of uniform linear transformations $\mathcal{L}$, it follows that

$$
\mathbf{X}^{r}=\sum_{i=1}^{q} \alpha_{i} \mathbf{X}_{i}^{r}
$$

Thus, if a $3 D$ view of an object can be represented as the weighted sum of views of other objects, its rotated view is a linear combination of the rotated views of the other objects with the same weights. Of course for an arbitrary $2 \mathrm{D}$ view that is a projection of a $3 \mathrm{D}$ view, a decomposition like (1) does not in general imply a decomposition of the rotated $2 \mathrm{D}$ views (it is a necessary but not a sufficient condition).

$2 D$ projections of $3 D$ objects 




Fig. 1. Learning an image transformation according to a rotation of three-dimensional cuboids from one orientation (upper row) to a new orientation (lower row). The 'test' cuboid (upper row right) can be represented as a linear combination of the two-dimensional coordinates of the three example cuboids in the upper row. The linear combination of the three example views in the lower row, using the coefficients evaluated in the upper row, results in the.correct transformed view of the test cuboid as output (lower row right). Notice that correspondence between views in the two different orientations is not needed and different points of the object may be occluded in the different orientations.

The question we want to answer here is, "Under which conditions the $2 \mathrm{D}$ projections of $3 \mathrm{D}$ objects satisfy equation (1) to (2)?" The answer will clearly depend on the types of objects we use and also on the projections we allow. We define:

$A$ set of $3 D$ views (of objects) $\left\{\mathbf{X}_{i}\right\}$ is a linear object class under $a$ linear projection $P$ if $\operatorname{dim}\left\{\mathbf{X}_{i}\right\}=\operatorname{dim}\left\{\mathbf{P X}_{i}\right\}$ with $\mathbf{X}_{i} \in \Re^{3 n}$ and $\mathbf{P} \mathbf{X}_{i} \in \Re^{p}$ and $p<3 n$

Note that the linear projection $P$ is not restricted to projections from $3 D$ to $2 D$, but may also "drop" occluded points. Now assume $\mathbf{x}=P \mathbf{X}$ and $\mathbf{x}_{i}=P \mathbf{X}_{i}$ being the projections of elements of a linear object class with

$$
\mathbf{x}=\sum_{i=1}^{q} \alpha_{i} \mathbf{x}_{i}
$$

Then $\mathbf{x}^{r}=P \mathbf{X}^{r}$ can be constructed without knowing $\mathbf{X}^{r}$ using $\alpha_{i}$ of equation 
(3) and the given $\mathbf{x}_{i}^{r}=P \mathbf{X}_{i}^{r}$ of the other objects

$$
\mathbf{x}^{r}=\sum_{i=1}^{q} \alpha_{i} \mathbf{x}_{i}^{r} .
$$

Figure 1 shows a very simple example of a linear object class and the construction of a new view of an object. Taking the 8 corners of a cuboid as features, a 3D view $\mathbf{X}$, as defined above, is an element of $\Re^{24}$; however, the dimension of the class of all cuboids is only 3 , so any cuboid can be represented as a linear combination of three cuboids. For any projection, that preserve these 3 dimensions, we can apply equations (3) and (4). The projection in figure 1 projects all non occluded corners orthographically onto the image-plane $\left(\mathbf{x}=P \mathbf{X} \in \Re^{14}\right)$ preserving the dimensionality. Notice that the orthographic projection of an exactly frontal view of a cuboid, which would result in a rectangle as image, would preserve 2 dimensions only, so equation (4) could not guarantee the correct result.

\section{$2.2 \quad$ Texture of $3 \mathrm{D}$ objects}

In this section we extend our linear space model from a representation based on the location of feature points only to textured feature points. The texture of a $3 \mathrm{D}$ object can be represented by a vector $\mathbf{t} \in \Re^{n}$, that is, by the texture values of its $n$ feature points. Since the texture or image irradiance of an object is in general a complex function of albedo, surface orientation and the direction of illumination, we have to distinguish different situations.

Let us first consider the easy case of objects all with the same identical texture: corresponding feature points of different objects have the same intensity or color. In this situation a single texture map (e.g. the reference image) is sufficient for a whole object class.

Next consider the situation in which the texture is a function of albedo only, that is independent of the surface normal. Then a linear texture class can be formulated in a way equivalent to equations (1) through (4). Assuming $\mathbf{t}_{i}$ being the texture maps of corresponding feature points of a class of objects. Then equation

$$
\mathbf{t}=\sum_{i=1}^{q} \beta_{i} \mathbf{t}_{i}
$$

with $\beta_{i}$ (different to $\alpha_{i}$ in equation (3)) implies for the "rotated" textures

$$
\mathbf{t}^{r}=\sum_{i=1}^{q} \beta_{i} \mathbf{t}_{i}^{r},
$$

assuming that the texture is independent of the surface orientation and the projection does not change the dimensionality of the texture space. In an application the coefficients $\alpha_{i}$ for the shape and coefficients $\beta_{i}$ for the texture can be computed separately. In face recognition experiments [4] the coefficients $\beta_{i}$ were already used as a rotation invariant representation of textures of faces. 


\section{An Implementation for Grey-Level Pixel Images}

We applied the linear class idea to grey-level images of human faces, each given in two orientations $\left(22.5^{\circ}\right.$ and $\left.0^{\circ}\right)$ in a resolution of 256 -by-256 pixels and 8 bit.

\subsection{Computation of the correspondence}

Our method assumes correspondence, and thus we have to find corresponding feature points in the two images and the associated spatial difference vector. For each prototype image separately, we computed the correspondence relative to a reference image, that is we found for every pixel location in the reference image, e.g. a pixel at the tip of the nose, the corresponding pixel location in the other images. This is in general a hard problem but here we need correspondence only between prototypical objects shown in the same pose, that is only between quite similar images. This makes it feasible to compute correspondence with automatic techniques such as optical flow algorithms. We use a coarse-to-fine gradient-based gradient method [7] and follow an implementation described in [8]. For every point $x, y$ in an image $I$, the error term $E=\sum\left(I_{x} \delta x+I_{y} \delta y-\delta I\right)^{2}$ is minimized for $\delta x, \delta y$, with $I_{x}, I_{y}$ being the spatial image derivatives and $\delta I$ the difference of intensity of the two compared images. The final result of this computation $(\delta x, \delta y)$ is used as an approximation of the spatial displacement of a pixel from one image to the other. For an image of $n$-by- $n$ pixels these displacements represent a correspondence field $\Delta \mathbf{x}$ of the image to a reference image with $\Delta \mathbf{x} \in \Re^{2 n^{2}}$.

\subsection{Projection of a test image into a linear shape and texture space}

First we compute the correspondence field $\Delta \mathbf{x}$ of the new test image to the reference image (which is in the same pose). Using the correspondence fields $\Delta \mathbf{x}_{i}$ of the other example images to the reference image and following equation (3), we computed the coefficients $\alpha_{i}$ by minimizing $\left\|\Delta \mathbf{x}-\sum_{i=1}^{q} \alpha_{i} \Delta \mathbf{x}_{i}\right\|^{2}$ with a SVD algorithm. We solved equation (5) for the coefficients $\beta_{i}$ assuming that the texture is a function of the albedo only. As a preliminary step the textures of all prototypical objects were mapped along the correspondence fields onto the reference image, so that all corresponding pixels in the prototypical images were mapped to the same pixel location in the reference image.

\subsection{Synthesis of the new image.}

The final step is image rendering. We compute the new texture and the new correspondence fields applying the computed coefficients $\beta_{i}$ and $\alpha_{i}$ to the examples given in the second orientation (equations (6) and (4)). The new image is then generated combining this texture and correspondence field. This is possible because both are given in the coordinates of the reference image. That means that for every pixel in the reference image we know the pixel texture value and 
1)
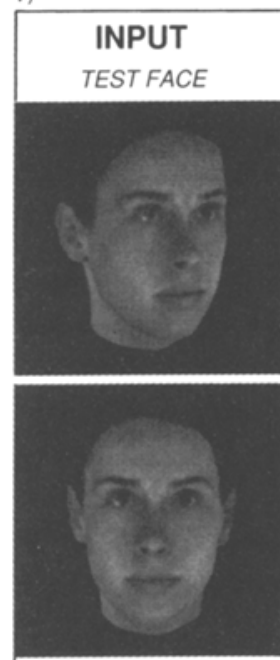

ROTATED TEST FACE

3)
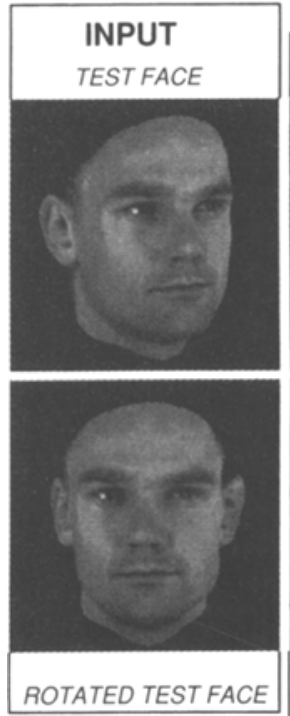
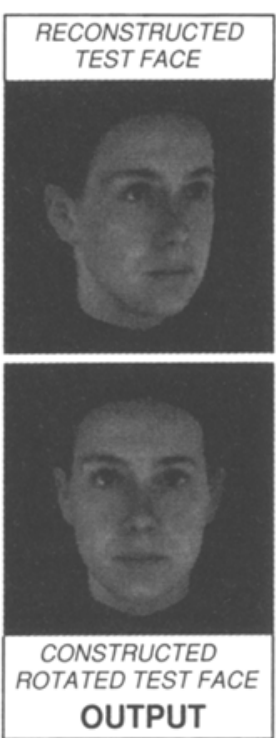

OUTPUT
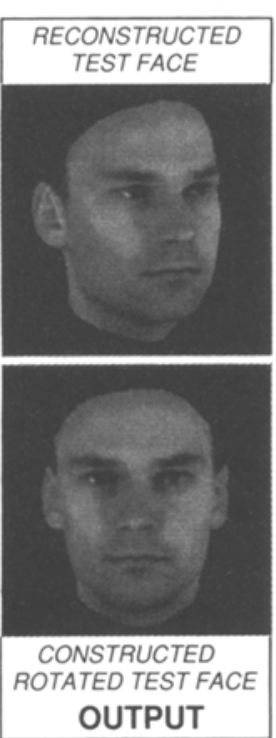

2)

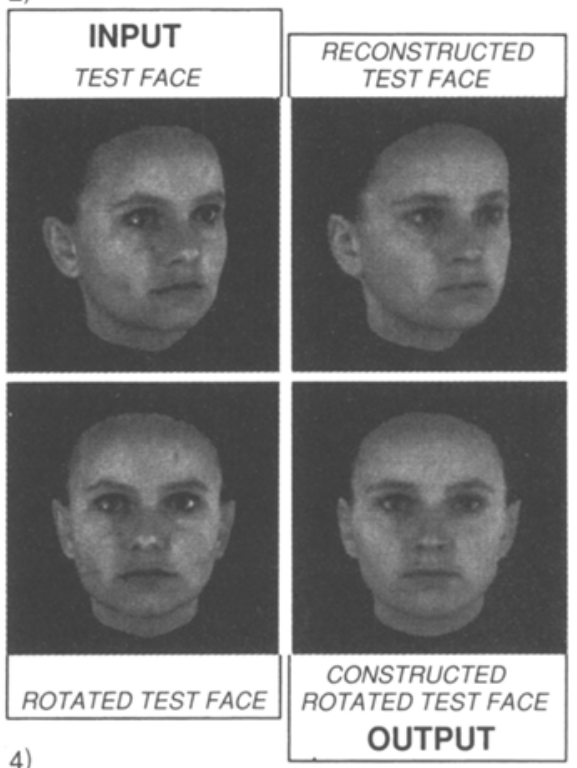

4)
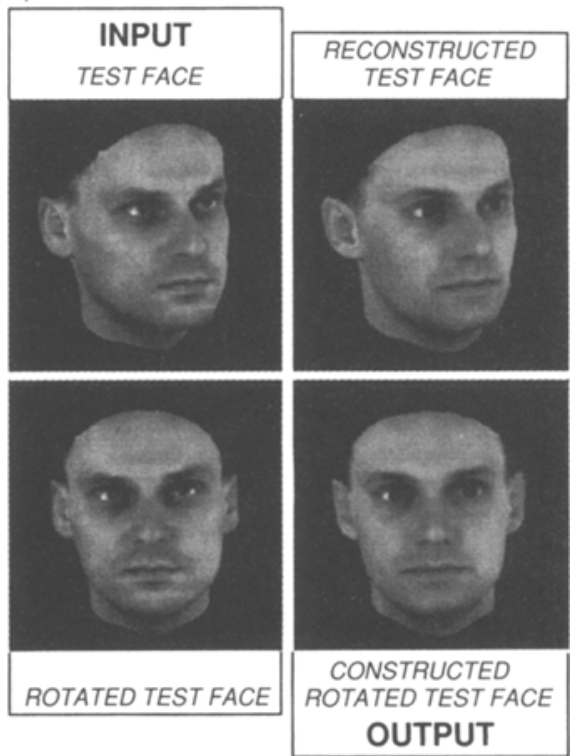

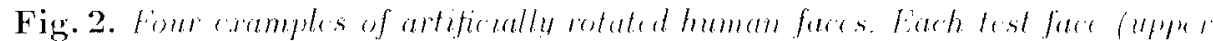

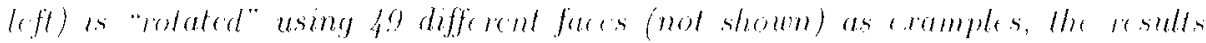

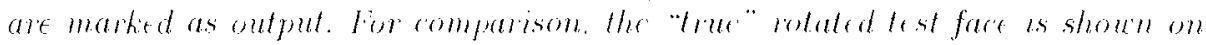
the lower left (this face was not used in the computation). The differenere betuest synthetic and real rotated face is due to the incomplete example set, sines the same difference can already be son in the reonstruction of the imput te st fact using the 4.9 erample fares (upper right). 
the vector pointing to the new location. The new location generally does not coincide with the equally spaced grid of pixels of the destination image. A commonly used solution of this problem is known as forward warping [9], which for every new pixel, uses the nearest three points to linearly approximate the pixel intensity.

\section{Is the Linear Class Assumption Valid for Human Faces?}

For man made objects, which often consist of cuboids, cylinders or other geometric primitives, the assumption of linear object classes seems natural. However, in the case of faces it is not clear how many example faces are necessary to synthesize any other face and in fact, it is unclear if the assumption of a linear class is appropriate at all. The key test for the linear class hypothesis in this case is how well the synthesized rotated face approximates the "true" rotated face. We tested our approach on a small set of 50 faces, each given in two orientations $\left(22.5^{\circ}\right.$ and $\left.0^{\circ}\right)$. Figure 2 shows four tests using the technique described earlier. In each case one face was selected as test face and the 49 remaining faces were used as prototypical examples. Each test face is shown on the upper left and the output image produced by our technique on the lower right, showing a rotated test face. The true rotated test face from the data base (not used in the computation) is shown on the lower left. We also show in the upper right the synthesis of the test face through the 49 example faces in the test orientation. This reconstruction of the test face should be understood as the projection of the test face into the shape and texture space of the other 49 example faces. A perfect reconstruction of the test face would be a necessary (not sufficient!) requirement for the claim that the 50 faces are a linear object class. Overall, the results are not perfect but, considering the small size of the sample set, the reconstruction is quite good. In our experiments, even in the cases were human observers judge the synthetic faces most different from the original face images, the synthetic images look like human faces and show clear characteristics of the target faces. The similarity of the reconstruction to the input test face suggests that an example set size of the order of hundred faces may be sufficient to construct a huge variety of different faces. We conclude that the linear object class approach may be a satisfactory approximation even for complex objects such as faces. On the other hand, it is also obvious that the reconstruction of every specific mole or wrinkle in a face requires an almost infinite number of examples. To overcome this problem, correspondence between images taken from different viewpoints could be used to map the specific texture on the new pose $[3,4]$.

\section{Discussion}

Linear combinations of linear drawing images of a single object have been already successfully used to create a new image of the same object [10]. Here we created a 
new grey-level image of an object using linear combinations of images of different objects of the same class. Given only a single image of an object, we are able to generate additional synthetic images of this object under the assumption that the "linear class" property holds. This is demonstrated not only for objects purely defined through their shape but also for smooth objects with texture.

This approach based on two-dimensional models does not need any depth information, so the sometime difficult step of generating three-dimensional models from two-dimensional images is superfluous. Since no correspondence is necessary between images representing objects in different poses and orientations, fully automated algorithms can be applied for the correspondence finding step. For object recognition tasks our approach has several implications. Our technique can provide additional artificial example images of an object when only a single image is given. Alternatively, the coefficients, which result from a decomposition of shape and texture into the prototypical shapes and textures, give us directly a representation of the object which is invariant under any 3D affine transformation and which may be used for recognition.

\section{References}

1. N. Troje and H. Bülthoff, "Face recognition under varying pose: The role of texture and shape," Vision Research in press.

2. T. Poggio and T. Vetter, "Recognition and structure from one $2 \mathrm{D}$ model view: observations on prototypes, object classes, and symmetries," A.I. Memo No. 1347, Artificial Intelligence Laboratory, Massachusetts Institute of Technology, 1992.

3. M. Jones and T. Poggio, "Model-based matching of line drawings by linear combination of prototypes," in ICCV'95, 1995.

4. D. Beymer and T. Poggio, "Face recognition from one model view," in ICCV 95, 1995.

5. T. Poggio and R. Brunelli, "A novel approach to graphics," Technical report 1354, MIT Media Laboratory Perceptual Computing Section, 1992.

6. D. Beymer, A. Shashua, and T. Poggio, "Example-based image analysis and synthesis," A.I. Memo No. 1431, Artificial Intelligence Laboratory, Massachusetts Institute of Technology, 1993.

7. J. Bergen, P. Anandan, K. Hanna, and R. Hingorani, "Hierarchical model-based motion estimation," in Proceedings of the European Conference on Computer Vision, (Santa Margherita Ligure, Italy), pp. 237--252, 1992.

8. J. Bergen and R. Hingorani, "Hierarchical motion-based frame rate conversion," technical report, David Sarnoff Research Center Princeton NJ 08540, 1990.

9. G. Wolberg, Image Warping. Los Alamitos CA: IEEE Computer Society Press, 1990.

10. S. Ullman and R. Basri, "Recognition by linear combinations of models.," IEEE Transactions on Pattern Analysis and Machine Intelligence, vol. 13, pp. 992-1006, 1991. 